Vol. 16, Núm. 2

Abr.-Jun. 2020. pp 62-65 doi: $10.35366 / 94534$

https://dx.doi.org/10.35366/94534

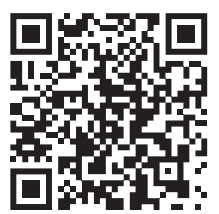

\title{
La traumatología deportiva ante COVID-19
}

\author{
Sports traumatology facing COVID-19
}

Fernando Rangel Gutiérrez*

\section{* Coordinador Comité de Traumatología del Deporte de la FEMECOT. Médico adscrito al Servicio de Artroscopia en la UMAA No. 90, IMSS, Torreón, Coahuila.}

Correspondencia: Dr. Fernando Rangel Gutiérrez. Calle Naranjos No. 109, Col. Torreón Jardín, C.P. 27200, Torreón, Coahuila, México, Correo electrónico: rangelferr@hotmail.com

\section{RESUMEN}

La traumatología deportiva pasa por un momento difícil ante esta pandemia del COVID-19. Siendo el deporte una actividad estrechamente relacionada con nuestra especialidad, hacemos algunas recomendaciones. Un estudio de ingeniería civil y deportes aerodinámicos realizado en Europa aconseja que para realizar deporte hay que mantener una distancia con los demás mínimo de 4 a 5 metros para caminar, 10 metros para correr o ciclismo lento y mínimo 20 metros para ciclismo rápido. Por otro lado, la Sociedad Española de Medicina del Deporte recomendó durante la fase de contingencia evitar zonas deportivas y realizar programas de mantenimiento físico en el propio domicilio. Para el reinicio de actividades es importante considerar para el deporte individual mantener las distancias con los demás, para el deporte en conjunto además de las distancias realizar actividades con grupos reducidos, limpieza constante del equipamiento y donde tengan la posibilidad de realizar pruebas diagnósticas a los jugadores e involucrados. Acerca de las cirugías en el preoperatorio, se recomienda realizar cuestionario, prueba para COVID-19 de 48 a 72 horas previo a cirugía, entre otros estudios. En medicina deportiva en la fase III de la pandemia se elimina la

\section{ABSTRACT}

Sports Traumatology is going through a difficult time in the face of this COVID-19 pandemic. Being the sport an activity closely related to our specialty we make some recommendations. A study of civil engineering and aerodynamic sports carried out in Europe advises that in order to do sport you must maintain a distance of at least 4 to 5 metres from others in order to walk, 10 meters for running or slow cycling and at least 20 meters for fast cycling. On the other hand, the Spanish Society of Sports Medicine recommended during the contingency phase to avoid sports areas and to carry out physical maintenance programs at home. For the restart of activities it is important to consider for the individual sport to maintain the distances with the others, for the sport as a whole besides the distances to carry out activities with reduced groups, constant cleaning of the equipment and where they have the possibility to carry out diagnostic tests to the players and involved. About the surgeries in the preoperative it is recommended to make questionnaire, test for COVID-19 of 48 to 72 hours previous to surgery among other studies. In sports medicine, phase III of the pandemic eliminates elective practice, scheduling of 
práctica electiva, programar lesiones recientes de extremidad inferior con incapacidad para el apoyo, cualquier padecimiento relacionado a infección, lesiones recientes de extremidad superior con incapacidad para mover activamente la articulación, padecimientos con lesiones musculares mayores, lo referente a luxaciones, fracturas y diferir todos los padecimientos con dolor agudo de extremidad inferior y superior con capacidad para apoyar y mover la articulación respectivamente.

Palabras clave: COVID-19, deporte, cirugía, preoperatorio, electiva. recent lower-extremity injuries with inability to support, any infection-related conditions, recent upper-extremity injuries with inability to actively move the joint, conditions with major muscle injuries, concerning dislocations, fractures and deferring all conditions with acute lower and upper extremity pain with the ability to support and move the joint respectively.

Keywords: COVID-19, sport, surgery, preoperative, elective.

\section{INTRODUCCIÓN}

La estrecha relación del deporte con nuestra especialidad y las lesiones derivadas de esta actividad conllevan medidas preventivas, además del momento adecuado para el manejo quirúrgico en esta pandemia del COVID-19. En este momento de medidas preventivas ante el COVID-19, en México la postura para los atletas y población en general es realizar actividad física regular con rutinas de ejercicios en casa para evitar riesgos de contagio y propagación de la enfermedad, así como también para disminuir riesgos de lesiones que puedan requerir atención médica y no saturar los sistemas de salud en estos momentos. En caso de realizar actividades físicas al exterior de casa, si el gobierno local lo permite, caminar con un mínimo de distancia entre otra persona de 4 a 5 metros, correr y ciclismo lento 10 metros y ciclismo rápido 20 metros.

Para el reinicio de actividades deportivas, esperando sea en las siguientes semanas, es importante considerar para el deporte individual mantener las distancias con los demás, para el deporte en conjunto, además de las distancias, realizar actividades con grupos reducidos, limpieza constante del equipamiento y donde tengan la posibilidad de realizar pruebas diagnósticas a los jugadores e involucrados.

Hay que diferir lo más posible en estos momentos cirugías electivas para evitar riesgos de contagio y propagación de la enfermedad. Sólo recomendamos realizar cirugías en medicina deportiva en padecimientos recientes con lesión y dolor agudo de extremidades inferiores con incapacidad para el apoyo y con limitación de la movilidad activa de la articulación en el caso de extremidades superiores, además de procesos infecciosos, lesiones musculares mayores, fracturas y luxaciones. Tratar de diferir las cirugías que ameriten una pronta rehabilitación en el postoperatorio y periodos prolongados de la misma, como en el caso de la reconstrucción del ligamento cruzado anterior y cirugías de hombro, tomando como consideración que en los servicios de rehabilitación en este momento hay restricciones. 


\section{Objetivo de la declaración de posición}

Disminuir los riesgos de propagación y contagio del COVID-19 del área médica y la población en general.

\section{MATERIAL Y MÉTODOS}

Criterios de búsqueda. Publicaciones acerca de COVID-19 en relación a medicina deportiva y traumatología.

Buscadores: PRIME PubMed, Google.

\section{RESULTADOS}

Se encontraron seis publicaciones relacionados con este tema. Existen pocas publicaciones del COVID-19 en relación a Medicina Deportiva y Traumatología por la reciente aparición de esta pandemia hasta este momento. Sólo se encontraron dos Guías de Procedimientos y Cirugía Electiva en Traumatología en Específico para medicina deportiva ante COVID-19, la de AAOS (American Academy of Orthopaedic Surgeons) y la de ESSKA (European Society of Sports Traumatology, Knee Surgery and Arthroscopy).

\section{DISCUSIÓN}

Existe controversia en la realización de actividad física dentro o fuera de casa ante el COVID-19, el pronunciamiento del Colegio Americano de Medicina Deportiva es el de realizar actividades físicas al aire libre, si el gobierno lo permite, de caminar o trotar alrededor del vecindario manteniendo la distancia de 6 pies con los demás, además de algún paseo en bicicleta. ${ }^{1}$ Un estudio de ingeniería civil y deportes aerodinámicos por la Universidad de Tecnología de Eindhoven y por KU Leuven en Bélgica aconseja mantener una distancia de al menos 4 a 5 metros para caminar, 10 metros para correr o ciclismo lento y mínimo 20 metros para ciclismo rápido, ${ }^{2}$ caso contrario a la recomendación que realizó durante el confinamiento la Sociedad Española de Medicina del Deporte en su país de realizar el programa de mantenimiento de aptitud física solamente en el propio domicilio. ${ }^{3}$ En países de Asia también se pronunciaron por realizar la actividad física solamente en casa y evitar la propagación de la enfermedad. ${ }^{4}$ Existen pocos colegios, sociedades o federaciones del deporte en el mundo que se han pronunciado en cuanto a este tema, aunque hay que tomar en cuenta las diferentes restricciones que existen en los gobiernos de cada país hacia la población ante el COVID-19. Nuestra postura es realizar actividad física preferentemente en casa, además de las recomendaciones de cada gobierno local, ante la incertidumbre de esta pandemia y la actual fase III de ésta. En caso de realizar actividades físicas fuera de casa, considerar las recomendaciones del estudio de Holanda y Bélgica. Para el reinicio de la actividad deportiva en las siguientes semanas considerar esta última recomendación de las distancias entre los demás, para el deporte en 
equipo es importante que el regreso sea en grupos reducidos, mantener el equipo y las instalaciones en constante limpieza, preferentemente realizar pruebas de COVID-19 a los jugadores y las personas involucradas con el equipo.

Es importante el entorno quirúrgico en la ortopedia y la traumatología deportiva, así como las medidas y equipos de protección personal durante la cirugía. En el manejo preoperatorio es recomendable realizar cuestionarios a los pacientes, pruebas de COVID-19 de 48 a 72 horas antes de la cirugía, considerar otras pruebas diagnósticas como TAC de pulmón, proteína $C$ reactiva y monitorización de la temperatura. En cuanto al postoperatorio, de ser posible utilizar videoconferencias y/o telemedicina para minimizar visitas repetitivas y limitar el desplazamiento del paciente. ${ }^{5}$ Respecto a las cirugías realizadas en medicina deportiva, la American Academy Orthopaedic Surgeons publicó recientemente su guía ante la pandemia COVID-19 donde hace recomendaciones de programación de cirugías electivas y el diferimiento de acuerdo a características de la patología en correlación a la fase en la que se encuentra la pandemia. ${ }^{6}$ En Méxi$\mathrm{co}$, podemos tomar como modelo esta guía y apegarla a los distintos protocolos de los sistemas de salud que se están realizando.

\section{CONCLUSIONES}

Evitar realizar actividades físicas y deportivas fuera de casa. Ante la fase III de la pandemia en México, es válido el diferimiento de la gran mayoría de cirugías electivas en medicina deportiva. Sólo es recomendable realizar cirugías en padecimientos con lesión y dolor agudo de extremidades con incapacidad para el apoyo y movilidad activa de la articulación, además de procesos infecciosos, lesiones musculares mayores, fracturas y luxaciones. Es probable que en los siguientes días aparezcan nuevas publicaciones de posturas y recomendaciones de las diferentes áreas de la medicina ante el desconocimiento aún de esta nueva entidad.

\section{BIBLIOGRAFÍA}

1. American College of Sports Medicine. Mantenerse activo durante la pandemia de coronavirus. Exercise is Medicine, 2020; Mar 26.

2. Thoelen J. Belgian-Dutch study: why in times of COVID-19 you should not walk/run/bike close behind each other. [April 7, 2020]. [Cited on may 13, 2020] In: Medium. Get Smart about what matters to you [Internet blog] Available in: https://medium.com/@jurgenthoelen/belgian-dutch-study-why-in-times-ofcovid-19-you-can-not-walk-run-bike-close-to-each-other-a5df19c77d08.

3. Manonelles P, Terreros JL, Romero S. Recomendaciones de la Sociedad Española de Medicina del Deporte, de la Agencia de Protección de la Salud en el Deporte y del Consejo General de Colegios Oficiales de Médicos de España en relación con el entrenamiento de deportistas en el estado de alarma decretado en España. 15 de Marzo de 2020. Disponible en: http://www.femede.es/documentos/ Recomendaciones_entrenamiento-COVID.pdf.

4. Chen P, Mao L, Nassis GP, Harmer P, Ainsworth BE, Li F. Coronavirus disease (COVID-19). The need to maintain regular physical activity while taking precautions. J Sport Health Sci. 2020; 9: 103-104.

5. Mouton C, Hirschmann M, Ollivier M, Seil R, Menetrey J. COVID-19-ESSKA Guidelines and Recommendations for Resuming Elective Surgery, European Society of Sports Traumatology, Knew Surgery \& Arthroscopy. 2020 (Last access may 13, 2020) file:///C:/Users/Ana\%20Maria\%20Mendoza/ Downloads/COVID-guidelines-QA.pdf.

6. Guy D, Bosco JA, Savoie FE. AAOS Guidelines for elective surgery during the COVID-19 pandemic. 2020. 